\title{
HIPERTENSÃO ARTERIAL NA GESTAÇÃO: avaliação da adesão ao tratamento
}

\author{
Élida Parreira MORAIS ${ }^{1}$ \\ Márcia Helena Cardoso Miranda PODESTÁ ${ }^{2}$ \\ Walnéia Aparecida de SOUZA ${ }^{2}$ \\ Eric Batista FERREIRA ${ }^{2}$
}

\begin{abstract}
${ }^{1}$ Residente Multiprofissional em Saúde da Família pela Universidade Federal de Alfenas. UNIFAL-MG. AlfenasMG.E-mail: elida.pmorais@yahoo.com.br

${ }^{2}$ Docentes da Universidade Federal de Alfenas-MG.
\end{abstract}

Recebido em: 10/03/2015 - Aprovado em: 12/08/2015 - Disponibilizado em: 30/10/2015

\begin{abstract}
RESUMO: O controle da pressão arterial durante a gestação é fundamental para que o desfecho desta seja o melhor possível. A não adesão terapêutica pode resultar em diversas consequências na saúde da gestante e do feto. Este trabalho teve como objetivo determinar a adesão ao tratamento medicamentoso de gestantes hipertensas de alto risco através da escala Morisky Medication Adherence Scale (MMAS-8) e relacionar com os caracteres sociodemográficos. Foi realizado um estudo transversal por meio de entrevista estruturada a pacientes gestantes hipertensas de alto risco, usuárias do Sistema Único de Saúde (SUS), atendidas em um ambulatório no Sul de Minas Gerais, entre maio e dezembro de 2013. A adesão foi determinada por meio da versão da escala MMAS-8. Foram consideradas aderentes aquelas pacientes com pontuação igual a 8 na MMAS-8. A prevalência encontrada da adesão terapêtica entre as gestantes foi de $18,4 \%$. Das entrevistadas, $68,4 \%$ apresentaram valores da pressão arterial sobre controle e não foi obtido grau de significância entre as variáveis de interesse e a adesão ao tratamento. Conclui-se que é necessário um reconhecimento mais amplo do problema da falta de adesão ao medicamento e uma vez identificado, estratégias simples deve ser implementadas na prática diária para melhorar a adesão.
\end{abstract}

Palavras-chave: Gestantes Hipertensas. Adesão ao Tratamento; Gestação de Alto Risco; Tratamento Medicamentoso; Sistema Único de Saúde.

\section{HYPERTENSION IN PREGNANCY: adherence to treatment assessment}

\begin{abstract}
The control of blood pressure during pregnancy is critical to its best possible outcome. The nonadherence to trearment can cause different consequences both to the mother and fetus's health. This paper has the objective to determine the adherence to drug treatment of high-risk hypertensive pregnant women by the Morisky Medication Adherence Scale (MMAS-8) and relate it to sociodemographic characters. Through of a cross-sectional study with structured interviews was performed with high-risk hypertensive pregnant patients of the Unified Health System (SUS) who were treated at a clinic in south Minas Gerais between May and December 2013. The adherence was determined by the MMAS- 8 scale. It was considered adherent those patients with score of 8 in the MMAS- 8 . The prevalence of adherence among the pregnant women was $18.4 \% .68 .4 \%$ of the interviewed women had high blood pressure levels under control. There was no degree of significance between the absorption and adherence to treatment variables. Therefore it was concluded that is necessary to have an understanding of the lack of medication adherence problem and once identified, simple strategies must be implemented in daily practice to improve adherence.
\end{abstract}

Keywords: Hypertensive Pregnant Women. Adherence to Treatment. High-Risk Pregnancy. Drug treatment. Public Health System. 


\section{INTRODUÇÃO}

As doenças crônicas têm uma representação significativa frente à população mundial, elas são apontadas como responsáveis por mais de $60 \%$ dos óbitos ocorridos no mundo. Dentre elas, a hipertensão arterial tem um papel de destaque acometendo um grande número de pessoas. Quando presente na gestação, a hipertensão mostra sérias complicações maternas e fetais incluindo piora do quadro hipertensivo, préeclâmpsia sobreposta, restrição do crescimento fetal, parto prematuro, descolamento prematuro da placenta e óbito fetal (FERRER et al., 2000; SIBAI, 2002; BRASIL, 2012).

As taxas de mortalidade materna no Brasil ainda são consideradas elevadas e a hipertensão arterial é uma das principais causas desse evento. Em 2012, o Ministério da Saúde publicou dados que apontam esta patologia como umas das principais causas da mortalidade materna, ocorrendo em cerca de $10 \%$ de todas as gestações (BRASIL, 2012).

O controle da pressão arterial durante a gestação é fundamental para que o desfecho desta seja o melhor possível. Atualmente empregam-se diferentes condutas para seu controle, sendo a mais utilizada a administração de agentes anti-hipertensivos (SIQUEIRA, F. et al., 2011).

Os principais medicamentos utilizados por via oral são $\alpha$-metildopa, $\beta$-bloqueador (atenolol e labetolol) e bloqueadores dos canais de cálcio (nifedipina e nicardipina). Nas emergências hipertensivas são utilizadas medicações intravenosas como hidralazina, labetolol, nitroglicerina e nitroprussiato de sódio (BARRILLEAUX; MARTIN JR, 2002; ROSENTHAL; OPARIL, 2002).

Dewulf et al. (2006) conceitua a adesão ao tratamento como o grau de concordância entre o comportamento de uma pessoa em relação às orientações do médico ou de outro profissional de saúde. O baixo grau de adesão pode afetar negativamente a evolução clínica do paciente e a sua qualidade de vida, constituindo-se em um problema relevante que pode trazer consequências pessoais, sociais e econômicas (WHO, 2003).

A adesão se caracteriza como um fenômeno sujeito de fatores relacionados às condições demográficas e sociais do paciente, à natureza da doença, às características da terapêutica, ao relacionamento do paciente com a equipe multiprofissional, bem como as características intrínsecas do próprio paciente. A importância de seguir o tratamento é fundamental para que o paciente consiga manter suas obrigações diárias e uma vida normal (MARINKER; SHAW, 2003; Sociedade Brasileira de Cardiologia, 2010).

A adesão à terapêutica medicamentosa tem sido investigada principalmente em condições crônicas. Assim, esse estudo apresentou como objetivo, a adesão ao tratamento da hipertensão em gestantes, uma vez que a não adesão pode resultar em 
diversas consequências na saúde da gestante e do feto, e, além disso, representa um tema pouco estudado.

\section{MATERIAIS E MÉTODOS}

\section{Aspectos Éticos}

Obedecendo a Resolução 196/96, que trata de pesquisas envolvendo seres humanos (BRASIL, 1996), todos os participantes do trabalho concordaram e assinaram o Termo de Consentimento Livre e Esclarecido. O projeto foi aprovado pelo Comitê de Ética em Pesquisa (CEP), parecer $n^{\circ} .564 .922$.

\section{População, local e período do estudo}

Foi realizado um estudo transversal por meio de entrevista estruturada, a pacientes gestantes hipertensas de alto risco, usuárias do Sistema Único de Saúde (SUS). Foram selecionadas 38 gestantes de alto risco, com diagnóstico confirmado de hipertensão arterial, atendidos em um ambulatório de um município do Sul de Minas Gerais, no período de maio a dezembro de 2014.

\section{Caracterização Sociodemográfica}

Por meio de um questionário, foram coletadas e analisadas as características demográficas e socioeconômicas que abrangeram sexo, idade, estado civil, raça, ocupação, escolaridade e renda familiar, além de dados relacionados a vida sexual, reprodutiva e ao pré-natal.

\section{Teste de Adesão ao Tratamento Medicamentoso}

A adesão ao tratamento foi determinada por meio da Escala de Morisky Medication Adherence Scale (MMAS- ${ }^{\circledR}$ ), traduzida e validada para o português por Oliveira Filho et al. (2012).

O (MMAS-8 ${ }^{\circledR}$ ) é composto por oito questões destinadas a medir a adesão à medicação. As primeiras sete são perguntas de caráter dicotômico sim / não, enquanto a última pergunta é respondida em uma escala de Likert de 5 pontos. Nas primeiras sete perguntas, um ponto é dado para cada resposta "NÃO", exceto para a pergunta número 5, onde um ponto é dado para a resposta "SIM". Para o item número 8 , um ponto é dado para o item "nunca" e ponto zero é dada para o item "sempre". A pontuação do MMAS- ${ }^{\circledR}$ é obtida pelo somatório das pontuações das 8 questões. A pontuação total obtida varia de 0-8 (SWEILEH, 2014). O grau de adesão terapêutica foi determinado de acordo com a pontuação resultante da soma de todas as respostas corretas: alta adesão (oito pontos), média adesão ( 6 a $<8$ pontos) e baixa adesão (< 6 pontos). Foram consideradas aderentes aquelas pacientes com pontuação igual a oito. 
Os valores de Pressão Arterial Sistólica (PAS) e Diastólica (PAD) foram obtidos pela equipe de enfermagem, momentos antes da entrevista e consulta. A definição de hipertensão na gravidez considera os valores absolutos de PA sistólica $\geq 140 \mathrm{mmHg}$ e/ou diastólica $\geq 90 \mathrm{mmHg}$ (Sociedade Brasileira de Cardiologia, 2010).

\section{Análise estatística}

Foi utilizado o teste Exato de Fisher, a $5 \%$ de significância ( $\mathrm{p}$ valor $\leq 0,05$ ), para verificar a associação entre variáveis de interesse: grau de adesão e faixa etária, escolaridade, ocupação e valor de pressão arterial.

\section{RESULTADOS}

Dentre as gestantes de alto risco atendidas no ambulatório, 23,4\% eram hipertensas, e destas, 38 participaram da pesquisa. A maioria das pacientes entrevistadas era casada, com menos de 30 anos e ensino médio completo. Quanto aos rendimentos, a maioria recebia entre um e dois salários mínimos e exerciam alguma profissão fora de casa (Tabela 1).

Tabela 1 - Caracterização sociodemográfica das gestantes hipertensas de alto risco $(\mathrm{n}=38)$

\begin{tabular}{|c|c|c|}
\hline Variáveis & n & $\%$ \\
\hline \multicolumn{3}{|l|}{ Idade } \\
\hline 20 a 30 & 20 & 52,64 \\
\hline 31 a 40 & 16 & 42,1 \\
\hline 41 a 46 & 2 & 5,26 \\
\hline \multicolumn{3}{|l|}{ Estado Civil } \\
\hline Casada & 27 & 71,05 \\
\hline Solteira & 2 & 5,26 \\
\hline União Consensual & 6 & 15,79 \\
\hline Divorciada & 3 & 7,89 \\
\hline \multicolumn{3}{|l|}{ Escolaridade } \\
\hline Fundamental Incompleto & 12 & 31,58 \\
\hline Fundamental Completo & 5 & 13,16 \\
\hline Médio Completo & 17 & 44,74 \\
\hline Superior & 4 & 10,53 \\
\hline \multicolumn{3}{|l|}{ Renda Familiar } \\
\hline Menor que 1 & 3 & 7,89 \\
\hline 1 a 2 & 24 & 63,16 \\
\hline 2 a 4 & 10 & 26,32 \\
\hline Maior que 4 & 1 & 2,63 \\
\hline \multicolumn{3}{|l|}{ Ocupação } \\
\hline Do lar & 17 & 44,74 \\
\hline Outros & 21 & 55,26 \\
\hline
\end{tabular}


$\mathrm{Na}$ história obstétrica pregressa, em relação ao número de gestações, $28,9 \%$ estavam grávidas pela primeira vez e $71,1 \%$ eram multigestas. Quanto aos partos e/ou abortos anteriores, $47,4 \%$ gestantes tinham partos anteriores e $15,8 \%$ nenhum aborto. A tabela 2 mostra os demais dados relacionados à vida sexual e reprodutiva das voluntárias.

Tabela 2 - Dados relacionados a vida sexual, reprodutiva e ao pré-natal

\begin{tabular}{|c|c|c|}
\hline & $\mathbf{n}$ & $\%$ \\
\hline \multicolumn{3}{|l|}{ Número de Gestações } \\
\hline Primigestas & 11 & 28,9 \\
\hline Multigestas & 27 & 71,1 \\
\hline \multicolumn{3}{|l|}{ Partos e/ou abortos } \\
\hline Nenhum parto e nenhum aborto & 11 & 28,9 \\
\hline Partos e nenhum aborto & 18 & 47,4 \\
\hline Partos e pelo menos um aborto & 7 & 18,4 \\
\hline Apenas abortos & 2 & 5.3 \\
\hline \multicolumn{3}{|l|}{ Partos Anteriores } \\
\hline Cesárea & 26 & 42,6 \\
\hline Parto normal & 35 & 57,4 \\
\hline \multicolumn{3}{|l|}{ Menarca (anos) } \\
\hline de 10 a 13 & 28 & 73,7 \\
\hline de 14 a 15 & 10 & 26,3 \\
\hline \multicolumn{3}{|l|}{ Sexarca } \\
\hline de 12 a 15 & 9 & 23,7 \\
\hline$\geq 16$ & 29 & 76,3 \\
\hline \multicolumn{3}{|l|}{ Metodo Contraceptivo } \\
\hline DIU & 1 & 2,6 \\
\hline Hormônio injetável & 3 & 7,9 \\
\hline Hormônio oral & 12 & 31,6 \\
\hline Tabela & 1 & 2,6 \\
\hline Preservativo masculino & 1 & 2,6 \\
\hline Nenhum & 20 & 52,6 \\
\hline
\end{tabular}

\subsection{Adesão ao tratamento}

A taxa de adesão terapêutica resultados estão demonstrados na Tabela 3. (MMAS-8 $=8$, alta adesão) foi de $18,4 \%$. Os 
Tabela 3 - Adesão ao tratamento medicamentoso de gestantes de alto risco, de acordo com os resultados obtidos pelo teste MMAS-8

\begin{tabular}{cccc}
\hline Adesão (pontos) & Classificação & n & $\%$ \\
\hline$\geq 8$ & Alta & 7 & 18,4 \\
$6<8$ & Média & 24 & 63,2 \\
$<6$ & Baixa & 7 & 18,4 \\
\hline Total & & 38 & 100 \\
\hline
\end{tabular}

\subsection{Variáveis associadas à adesão ao}

\section{tratamento}

$\mathrm{Na}$ investigação da associação entre a características sociodemográficas, não houve diferenças estatisticamente significativas. Os resultados encontram-se na tabela 4.

adesão ao tratamento medicamentoso e as

Tabela 4 - Adesão terapêutica e características sociodemográficas das gestantes

\begin{tabular}{|c|c|c|c|c|c|c|c|}
\hline \multirow{2}{*}{ Variável } & \multicolumn{2}{|c|}{ Alta adesão } & \multicolumn{2}{|c|}{ Média adesão } & \multicolumn{2}{|c|}{ Baixa adesão } & \multirow{2}{*}{$p$-valor } \\
\hline & $\mathbf{n}$ & $\%$ & $\mathbf{n}$ & $\%$ & $\mathbf{n}$ & $\%$ & \\
\hline \multicolumn{8}{|l|}{ Idade } \\
\hline Até 30 anos & 2 & 10,5 & 12 & 63,2 & 5 & 26,3 & 0.3341 \\
\hline$\geq 31$ anos & 5 & 26,3 & 12 & 63,2 & 2 & 10,5 & \\
\hline \multicolumn{8}{|l|}{ Escolaridade } \\
\hline Fundamental Incompleto & 2 & 16,7 & 8 & 66,7 & 2 & 16,7 & 1 \\
\hline Fundamental Incompleto & 5 & 19,2 & 16 & 61,5 & 5 & 19,2 & \\
\hline \multicolumn{8}{|l|}{ Renda Familiar } \\
\hline Até 2 salários & 5 & 18,5 & 17 & 63 & 5 & 18,5 & 1 \\
\hline$>2$ salários & 2 & 18,2 & 7 & 63,6 & 2 & 18,2 & \\
\hline \multicolumn{8}{|l|}{ Ocupação } \\
\hline Do lar & 2 & 11,8 & 12 & 70,6 & 3 & 17,6 & 0,7263 \\
\hline Outros & 5 & 23,8 & 12 & 57,1 & 4 & 19 & \\
\hline
\end{tabular}

\subsection{Fatores que afetam a não adesão}

$\mathrm{Na}$ análise individual dos critérios presentes no MMAS-8, nove participantes $(23,7 \%)$ relataram que às vezes se esqueciam de tomar seus medicamentos antihipertensivos e 26,3\% afirmaram já ter parado de tomar ou diminuído a dose do medicamento por se sentir pior com seu uso. Esses e os demais resultados encontram-se na tabela 
Tabela 5 - Comportamento da adesão ao tratamento medicamentoso através de auto-relato das gestantes hipertensas de alto risco pela escala MMAS-8.

\begin{tabular}{|c|c|}
\hline Item & $\frac{\text { SIM }}{\mathrm{n}(\%)}$ \\
\hline 1) Você às vezes esquece de tomar os seus remédios para pressão? & $9(23,7)$ \\
\hline $\begin{array}{l}\text { 2) Nas duas últimas semanas, houve algum dia em que você não tomou seus remédios para pressão } \\
\text { alta? }\end{array}$ & $6(15,8)$ \\
\hline $\begin{array}{l}\text { 3) Você já parou de tomar seus remédios ou diminuiu a dose sem avisar seu médico porque se sentia } \\
\text { pior quando os tomava? }\end{array}$ & $10(26,3)$ \\
\hline 4) Quando você viaja ou sai de casa, às vezes esquece de levar seus medicamentos? & $1(2,6)$ \\
\hline 5) Você tomou seus medicamentos para pressão alta ontem? & $36(94,7)$ \\
\hline 6) Quando sente que sua pressão está controlada, você às vezes para de tomar seus medicamentos? & $4(10,5)$ \\
\hline 7) Você já se sentiu incomodado por seguir corretamente o seu tratamento para pressão alta? & $14(36,8)$ \\
\hline \multicolumn{2}{|l|}{$\begin{array}{l}\text { 8) Com que frequência você tem dificuldades para se lembrar de tomar todos os seus remédios para } \\
\text { pressão? }\end{array}$} \\
\hline Nunca & $22(57,9)$ \\
\hline Quase Nunca & $7(18,4)$ \\
\hline Às Vezes & $7(18,4)$ \\
\hline Frequentemente & $1(2,6)$ \\
\hline Sempre & $1(2,6)$ \\
\hline
\end{tabular}

\subsection{Pressão Arterial e adesão}

Das pacientes entrevistadas, $68,4 \%$ (n=26) apresentaram valores de pressão arterial sob controle. A tabela 6 mostra a relação da pressão arterial e a adesão ao tratamento medicamentoso; não houve diferença significativa entre as duas variáveis $(\mathrm{p}$-valor $=0,3908)$.

Tabela 6 - Relação dos graus de adesão terapêutica com o controle e os valores médios da PA.

\begin{tabular}{ccccccc}
\hline & & \multicolumn{2}{c}{ PA controlada } & PA não controlada & p-valor \\
\cline { 3 - 6 } Adesão (pontos) & Classificação & $\mathbf{n}$ & $\mathbf{\%}$ & $\mathbf{n}$ & \% & \\
\hline$\geq 8$ & Alta & 5 & 20 & 2 & 15,4 & \\
$6<8$ & Média & 17 & 68 & 7 & 53,8 & 0.3908 \\
$<6$ & Baixa & 3 & 12 & 4 & 30 & \\
\hline Total & & 25 & 100 & 13 & 100 \\
\hline
\end{tabular}

$\mathrm{PA}=$ Pressão Arterial

\subsection{Tratamento Farmacoterapêutico}

Com relação aos medicamentos apresentou-se com maior relevância $(85,4 \%)$ seguido da nifedipina $(7,3 \%)$, pindolol $(4,9 \%)$ e hidralazina $(2,4 \%)$. A monoterapia foi prescritos durante a gestação, a alfa-metildopa 
predominante $(94,7 \%)$, sendo que a maioria $(89,5 \%)$ utilizava de 2 a 3 vezes ao dia.

\section{DISCUSSÃO}

Os resultados deste trabalho mostram que a maioria das gestantes hipertensas de alto risco não adere ao tratamento medicamentoso independentemente da idade, ocupação, grau de escolaridade e renda familiar. Do total das entrevistadas, 34,3\% apresentaram pressão arterial (PA) não controlada, um número expressivo uma vez que entre as doenças maternas que ocorrem, a hipertensão induzida pela gravidez no período gravídico, é considerada uma das que mais efeitos nocivos provocam no organismo materno, fetal e neonatal (CHAIM; OLIVEIRA; KIMURA, 2008).

O presente estudo aponta uma adesão ao tratamento medicamentoso de apenas $18,4 \%$ das gestantes hipertensas de alto risco. A adesão ao tratamento destas pacientes é importante para a sobrevida fetal e materna; manutenção de peso fetal adequado ao nascimento; baixa incidência de complicações e prevenção de eclâmpsia (COSTA, 2005).

Livingston et al.(2003) relatam que estas gestantes devem receber tratamento antihipertensivo agressivo e avaliações frequentes de bem-estar materno e fetal, recomendações de mudanças no estilo de vida e monitorização rígida no pós-parto, pois o manejo cuidadoso ante-parto, intraparto e pós-parto pode reduzir a morbidade e a mortalidade.

Não houve relação estatisticamente significativa entre as características sociodemográficas como idade, ocupação, escolaridade, renda familiar e o grau de adesão. Por exemplo, gestantes com mais de 30 anos apresentaram maior adesão que as mais jovens; da mesma forma, as que tinham uma ocupação fora de casa apresentaram maior adesão que as gestantes do lar, entretanto estas diferenças não foram estatisticamente significativas. Cohen et al. (2012) encontrou em seu estudo que a variável idade foi considerada como fator relacionado à adesão e que os pacientes mais velhos são mais aderentes, considera-se que haja associação direta entre aumento da idade e maior envolvimento com a problemática de saúde, o que deveria repercutir em maior controle da doença. Uma hipótese para tais achados é a maior sensibilização dessas gestantes para os riscos do uso de medicamentos, seja por maior acesso à informação ou por experiências negativas durante gestações anteriores.

Segundo manual do Ministério da Saúde (2008b), a adesão é um processo dinâmico e multifatorial, que inclui corresponsabilização entre a equipe de saúde e o sujeito. Não se trata de um ato de obediência ou conformação da pessoa, mas sim um processo de negociação que tem por 
objetivo favorecer a autonomia e o autocuidado.

De uma maneira geral, a taxa de adesão ao tratamento anti-hipertensivo é não é alta, a dificuldade em aderir ao tratamento medicamentoso é um problema constante, pois interfere na resposta terapêutica desejada. Trabalhos anteriores têm mostrado baixa adesão de hipertensos ao tratamento farmacológico (OBRELI-NETO, 2010; DOSSE et al., 2009; SPINATO; MONTEIRO; SANTOS, 2010).

Em relação à taxa de pacientes aderentes ao tratamento, este trabalho apresentou valores semelhantes ao de Oliveira et al.(2012), em que apenas 19,2\% das pacientes foram consideradas aderentes ao tratamento medicamentoso. No trabalho de Langaro e Santos (2014) foi encontrado $17 \%$ de adesão ao tratamento em gestantes que realizaram tratamento de 3 a 5 meses na Unidade de Saúde.

Para evidenciar os fatores que mais prejudicaram a adesão, foi feita a análise individual dos critérios presentes no MMAS8. O resultado revelou que os itens "Você já se sentiu incomodado por seguir corretamente o seu tratamento para pressão alta?" e "Você já parou de tomar seus remédios ou diminuiu a dose sem avisar seu médico porque se sentia pior quando os tomava?", obtiveram menor pontuação.

No trabalho de Langaro e Santos (2014), as gestantes relataram que, quanto maior o período de tratamento, maior a sensação de cansaço e desgaste ocasionado pelo investimento de tempo, esforço e disponibilidade emocional para as consultas, exames e controles, bem como para as mudanças de hábitos de vida.

A maioria $(94,7 \%)$ das participantes do estudo fazia uso de um único medicamento anti-hipertensivo. A monoterapia deveria ser um fator positivo para adesão ao tratamento, entretanto, o esquema posológico mais prevalente era de 2 a 3 vezes ao dia, possivelmente este foi um fator relevante para a baixa adesão. Trabalhos anteriores mostram que a diminuição do número de medicamentos utilizados e a diminuição da freqüência das dosagens, de preferência uma única dose diária, são importantes fatores que levam à adesão ao tratamento (RUDD apud ARAÚJO; GARCIA, 2006). O horário da tomada dos medicamentos também é um fator que intervém na adesão. Assim, ao correlacionar o horário das dosagens com atividades da rotina diária, que sirvam de lembretes, há melhora no nível de adesão.

Outro ponto relevante é o fato da hipertensão ser uma doença silenciosa, e ao fazer uso do medicamento, o paciente poderia sentir os efeitos adversos e por isso, interromper seu uso, reduzindo a taxa de adesão(ELLIOTT, 2008).

A alfa-metildopa foi o medicamento mais prescrito para as gestantes, resultado que está de acordo com as VI Diretrizes 
Brasileiras de Hipertensão. Este fármaco é referido como o preferido por ser o mais bem estudado e não haver evidência de efeitos deletérios para o feto.

No presente trabalho não foi possível determinar se o baixo valor da adesão ao tratamento farmacológico interferiu no controle da pressão arterial das gestantes. A correlação entre adesão à terapêutica medicamentosa e o controle da PA tem sido avaliada em vários estudos, sendo que em alguns deles a correlação tem sido positiva, encontrando-se melhor nível de controle da PA nos pacientes aderentes (OLIVEIRA et al, 2012), enquanto em outros não houve correlação estatisticamente significante (STRELEC; PIERIN; MION JR, 2003; PLASTER, 2006;).

\section{CONCLUSÃO}

A adesão ao tratamento medicamentoso em gestantes hipertensas de alto risco usuárias do SUS do município foi baixa. Foi possível detectar que fatores como a necessidade do uso diário de medicamento assim como a frequiência das dosagens, contribuíram para este resultado. Outro fator que se destacou foi o aparecimento de sinais e

\section{BIBLIOGRAFIA}

BARRILLEAUX, P.S.; MARTIN JR, J.N. Hypertension therapy during pregnancy. sintomas desagradáveis e incidência de efeitos colaterais.

A partir destes resultados encontrados, sugere-se que os serviços de saúde promovam a educação continuada dos profissionais de saúde com enfoque na comunicação com o paciente para fortalecer a importância da adesão ao tratamento medicamentoso. Acredita-se que a educação das gestantes hipertensas seja o melhor caminho para reduzir a taxa de mortalidade materna no Brasil e diminuir as situações de risco para o feto.

Foi observado também a existência de um reduzido número de estudos sobre a adesão terapêutica durante a gestação o que limita a comparação de achados, no entanto, os baixos níveis de adesão aqui observados indicam uma necessidade de aprofundamento no tema. A realização de outros estudos que permitam dimensionar o impacto da não adesão durante a gestação e suas causas, a investigação aprofundada de fatores socioeconômicos, bem como a relação entre a adesão terapêutica e o acompanhamento prénatal, pode esclarecer vários aspectos que atualmente comprometem ou otimizam a efetividade de diversos tratamentos prescritos durante

a gestação.
Clinical Obstet Gynecol. v. 45, p. 22-34, 2002 
Brasil (2008b). Ministério da Saúde.

Secretaria de Vigilância em Saúde. Programa

Nacional de DST e Aids. Manual de adesão

ao tratamento para pessoas vivendo com

HIV e Aids. Brasília, DF: Editora do

Ministério da Saúde.

BRASIL. Ministério da Saúde. Boletim

1/2012 - Mortalidade Materna no Brasil.

Brasília: Ministério da Saúde; 2012.

Disponível em:

http://portalsaude.saude.gov.br/portalsaude/in dex.cfm?portal=pagina.visualizarTexto\&cod

Conteudo $=6403 \&$ codModuloArea $=783 \&$ cha

$\underline{\text { mada=boletim- } 1 / 2012 \text { - } \_ \text {-mortalidade- }}$

materna-no-\%20\%20brasil. Acesso em 04 Jul

2013.

CHAIM, S. R. P.; OLIVEIRA, S. M. J. V;

KIMURA, A. F. Hipertensão Arterial na

Gestação e Condições Neonatais ao

Nascimento. Acta Paul Enferm. v. 21, n. 1,

p. 53-8, 2008.

COHEN, M. J. et al. Predictors of medication adherence postdischarge: the impact of patient age, insurance status, and prior adherence. J Hosp Med. v. 7, n.6, p.470-5, 2012.

COSTA, S.H.M. et al. Hipertensão Crônica E Complicações Na Gravidez. Revista da

\section{Sociedade de Cardiologia do Rio Grande}

do Sul. v. 15, n. 5, 2005.

DEWULF, N.L.S. et al. Adesão ao tratamento medicamentoso em pacientes com doenças gastrintestinais crônicas acompanhados no ambulatório de um hospital universitário. Revista Brasileira de Ciências Farmacêuticas. v.42, n. 4, p. 57584, 2006.

DOSSE, C. et al. Fatores associados à não adesão dos pacientes ao tratamento de hipertensão arterial. Rev Latino-am Enfermagem. v. 17, n. 2, p. 201-6, 2009.

ELLIOTT, J. W. What Factors Contribute to the Inadequate Control of Elevated Blood Pressure? The Journal of Clinical Hypertension. v. 11, n. suppl. 1, p. 20-26, 2008.

FERRER, R. et al. Management of mild chronic hypertension during pregnancy: A Review. Obstet Gynecol. v. 96, n. 5, p.84960, 2000.

GIROTTO, E. et al. Prevalência de fatores de risco para doenças cardiovasculares em hipertensos cadastrados em unidade de saúde da família. Acta Scientiarum. Health

Sciences. v. 31, n. 1, p. 77-82, 2009. 
LANGARO, F.; SANTOS, A. H. Adesão ao tratamento gestantes de alto risco. Psicol. cienc. prof. v. 34, n. 3, p. 625-642, 2014.

LIVINGSTON, J.C.; MAXWELL, B.D.; SIBAI, B.M. Chronic Hypertension in Pregnancy. Minerva Ginecol. v.55, n. 1, p. 1-13, 2003.

MARINKER, M.; SHAW J. Not to be taken as directed: putting concordance for taking medicines into practice. BMJ; v. 326, p. 34849, 2003.

MORISK, D.E. et al. Predictive validity of a Medication Adherence Measure in an outpatient setting. J Clin Hypertens. v. 10, n. 5, p. 348-54, 2008.

OBRELI-NETO, P. R. et al. Fatores Interferentes na Taxa de Adesão à Farmacoterapia em Idosos Atendidos na Rede Pública de Saúde do Município de Salto Grande - SP, Brasil. Rev Ciênc Farm Básica Apl. v. 31, n. 3, p. 229-233, 2010.

OLIVEIRA FILHO, A. D. et al. Aderência autorreferida a medicamentos prescritos durante a gestação. Rev Bras Ginecol

Obstet. v. 34, n. 4, p. 147-52, 2012.

OLIVEIRA-FILHO, A. D. et al. Relação entre a Escala de Adesão Terapêutica de Oito Itens de Morisky (MMAS-8) e o Controle da
Pressão Arterial. Arq Bras Cardiol. v. 99, n. 1, p. 649-658, 2012.

\section{PLASTER W. Adesão ao tratamento da} hipertensão arterial por idosos usuários da unidade básica de saúde Princesa Isabel em Cacoal-RO. 2006. 89f. Dissertação. Convênio Rede Centro-Oeste (UnB, UFG, UFMS), Goiânia; 2006.

ROSENTHAL, T.; OPARIL, S. The effect of antihypertensive drugs on the fetus. J Hum Hypertens. v. 16, p. 293-8, 2002. RUDD, P. Clinicians and patients with hypertension: unsettled issues about compliance. American Heart Journal In: ARAÚJO, G.B.S; GARCIA, T.R. Adesão ao Tratamento Anti-Hipertensivo: Uma Análise Conceitual. Revista Eletrônica de

Enfermagem. v. 8, n. 2, p. 259-272, 2006.

SIBAI, B. Chronic hypertension in pregnancy. ObstetGynecol. v. 100, n. 2, p. 369-77, 2002.

SIQUEIRA, F. et al. Medicamentos antihipertensivos na gestação e puerpério. Com. Ciências Saúde. v. 22, n. Sup 1, p.S55-S68, 2011.

Sociedade Brasileira de Cardiologia. VI diretrizes Brasileiras de Hipertensão Arterial. Arq Bras Cardiol. v. 95, p. 1-5, 2010. 
SPINATO, I.L.; MONTEIRO, L.Z.;

SANTOS, Z.M.S.A. Adesão da pessoa

hipertensa ao exercício físico - uma proposta

educativa em saúde. Texto contexto -

enferm. Florianópolis, v. 19, n. 2, p. 256-

264, Abr./Jun. 2010.

STRELEC, M. A, A. M.; PIERIN, A. M. G.;

MION JR, D. A influência do conhecimento

sobre a doença e a atitude frente à tomada

dos remédios no controle da hipertensão.

Arq Bras Cardiol. v. 81, n. 4, p. 343-8,

2003.

SWEILEH, W.M. et al. Influence of patients'

disease knowledge and beliefs about

medicines on medication adherence: findings

from a cross-sectional survey among patients

with type 2 diabetes mellitus in Palestine.

BMC Public Health. v. 14, n. 94, 2014.

\section{WORLD HEALTH ORGANIZATION.}

Adherence to long-term therapies: evidence

for action, Switzerland: Publications, 2003. 